\title{
SUPPLEMENT
}

For reprint orders, please contact: reprints@futuremedicine.com

\section{Nodular histiocytic/mesothelial hyperplasia as consequence of chronic mesothelium irritation by subphrenic abscess}

\author{
Daniela Cabibi', Giorgio Lo lacono², Francesco Raffaele², Salvatore Dioguardi², \\ Sabrina Ingrao', Antonio Pirrotta', Federica Fatica² \& Massimo Cajozzo ${ }^{*, 2}$
}

Purpose: Pleural nodular histiocytic/mesothelial hyperplasia is a nodular histiocytic/ mesothelial proliferation, often delimiting cystic cavities, due to irritation by a pulmonary noxa. Case report results: The patient had right pleural parietal and diaphragmatic thickness, with pleural effusion, without lung alterations. He previously underwent left hemicolectomy and liver resection, due to a diverticulitis and a liver histiocytes-rich abscess. Video-assisted thoracoscopy biopsy showed a double population of reactive mesothelial cells and histiocytes. Conclusion: Nodular histiocytic/mesothelial hyperplasia represents a potential pitfall for pathologists. Immunohistochemistry is crucial for the differential diagnosis with some malignancies. We suggest that in our patient, a chronic mesothelium inflammation happened by transdiaphragmatic involvement as a consequence of the liver abscess. Some pathogenetic mechanisms are hypothesized.

Nodular histiocytic/mesothelial hyperplasia (NHMH) is a benign localized alteration, first described in 1975 by Rosai in the hernia sac [1]. Few pulmonary cases have been reported in literature [2-6]. Sometimes it has been reported in the pericardium [7,8] or presenting as an inguinal mass [9]. The 'mesothelial/monocytic incidental cardiac excrescence', first described by Weinot et al. in 1994 [10] is now considered a similar lesion to NHMH [11].

It consists of a reactive proliferation of histiocytes and mesothelium secondary to chronic irritation and it has been observed in pleura-damaging processes, such as pneumothorax [5], or as consequence of cardiac catheterization, inflammation, mechanical or tumor stimulation [11].

The rarity of NHMH and the moderate cytological atypia often present, make this lesion difficult to diagnose. It can be easily confused with primary mesothelial lesions and neoplasms such as adenocarcinomas, granulosa cell tumors or Langerhans' histiocytosis.

We report a case of pleural NHMH in a patient with a subphrenic abscess, in which no pulmonary pathogenic noxa was evident. We hypothesize a transdiaphragmatic chronic irritation as a pathogenetic mechanism underlying NHMH.

\section{Case report}

A 57-year-old man presented to our department in June 2014, due to the presence of a right pleural effusion with undefined diagnosis. At admission he had no fever, infections or history of exposure to asbestos and other dust. Shortness of breath after moderate exertion was noted, blood pressure was $130 / 85 \mathrm{mmHg}$ and heart rate $77 \mathrm{bpm}$ with normal sinus rhythm. Physical examination showed abolition of breath sounds and fremitus on all fields on the right side and dullness plexus. The patients drug history was negative. Due to the onset of pleural effusion and dyspnea, the patient

\section{KEYWORDS}

- mesothelial hyperplasia

- pleural effusion • VATS 
SUPPLEMENT Cabibi, Lo lacono, Raffaele et al.
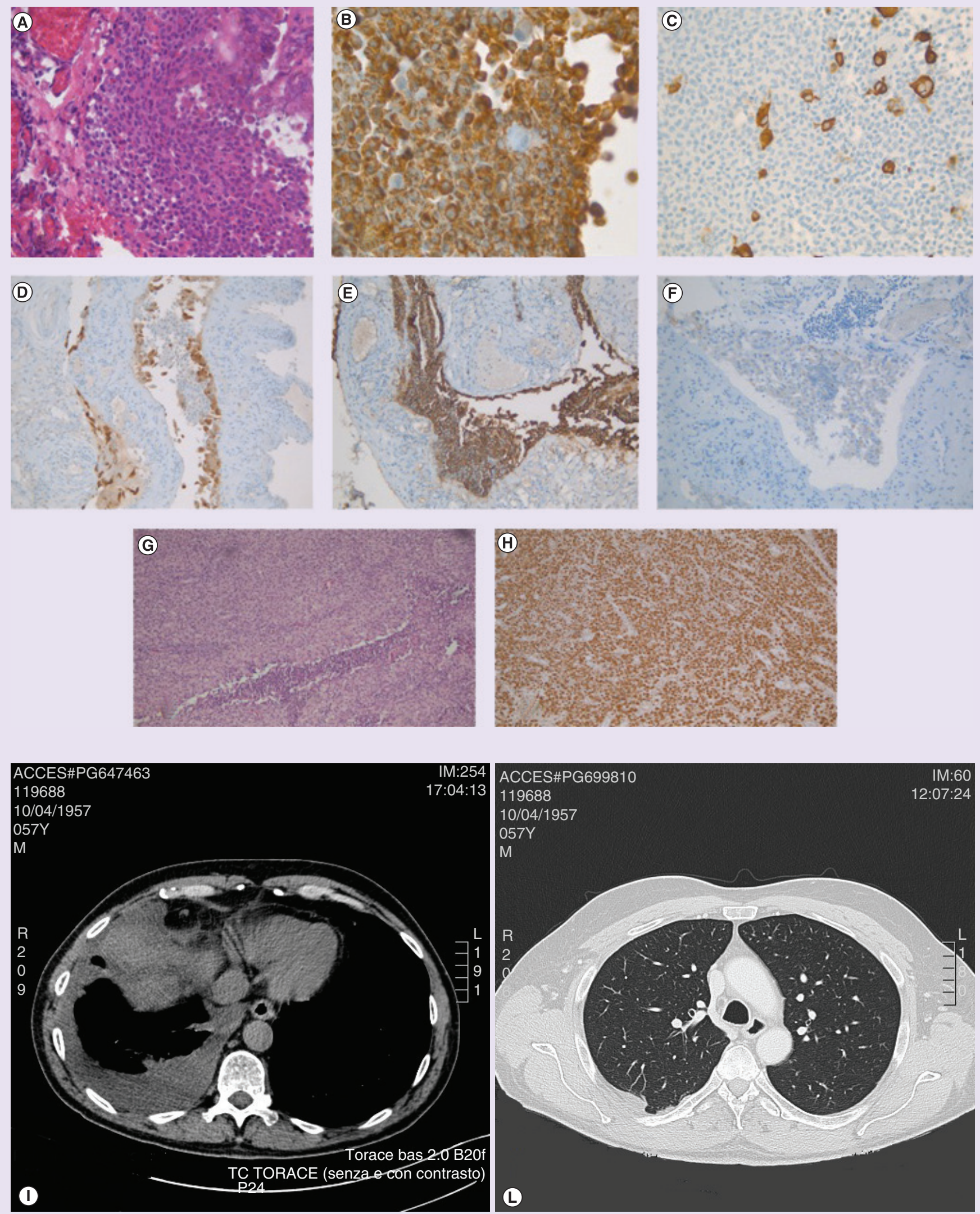
Figure 1. Histological examination and CT scan (see facing page). (A-F) Pleural biopsy: cystic cavitations covered by proliferations composed of a double population consisting of 'sheets' of histiocytes with grooved and slightly irregular nucleus, positive for CD68 (B) and cuboidal or low columnar mesothelial cells, isolated or multilayered, with moderate cytologic atypia, positive for calretinin (C \& D), HBME1 (E) and negative for EMA (F); (G \& H) liver abscess with an intense inflammatory infiltrate with sheets of histiocytes positive for CD68 immunostaining; (I-L): (I) right pleural effusion and mild irregular thickness on preoperative TC - (L) no progression of disease after 1-year TC follow-up. (A) Hematoxylin-eosin, original magnification 400x; (B) CD68 immunostaining; original magnification 640x; (C \& D) calretinin immunostaining; original magnification: (C) 400x; (D) 200x; (E) HBME1 immunostaining; original magnification 200x; (F) EMA immunostaining; original magnification 200x; (G) hematoxylin-eosin, original magnification 100x; (H) CD68 immunostaining; original magnification 100x; (I) Preooperative TC; (L) 1-year follow-up TC.

underwent thoracentesis. Pleural fluid cytology did not show any kind of atypical cells.

Because of the persistence of pleural effusion and dyspnea the patient underwent videoassisted thoracoscopy (VATS) for biopsy. The procedure showed multiple lesions on the right parietal and diaphragmatic pleura, partly nodular, partly similar to linear ulcerations, of which were biopsy samples were taken (12 samples were taken from the pleural and subpleural fat in three different sites).

The histological examination of the pleural biopsies showed frustules of edematous and congested connective tissue, focally covered by pleura, in which cystic cavitations were present. They were covered by proliferations composed of a double cell population. This consisted of 'sheets' of histiocytes with grooved and slightly irregular nucleus, with some giant cells and of cuboidal or low columnar mesothelial cells, isolated or, more often, multilayered, with moderate cytologic atypia. The histiocytes stained positvely for CD68 and negatively for CD1a and S100. The mesothelial cells stained positively for calretinin, HBME1, D2-40, CK AE1/AE3, CK5/6, WT1, vimentin and negatively for EMA and P53 (Figure 1A-F). Low proliferation index $(\mathrm{Ki} 67=5-10 \%)$.

There was an absence of mitosis, necrosis and stromal invasion, both in the visceral and in the parietal subpleural tissue. Histological diagnosis was therefore 'NHMH'.

Three months earlier (March 2014) during hospitalization for general malaise, the patient underwent a thoracoabdominal CT scan without contrast, which revealed an hepatic subcapsular lumpy lesion, with a diameter of approximately $4 \mathrm{~cm}$ and to a colonoscopy, which showed sigmoid substenosis and diverticulitis. The patient then underwent a left hemicolectomy and a resection of VI, VII and VIII hepatic segment (resected of $8 \times 8 \times 6 \mathrm{~cm}$ ). Histological examination of the resected colonic segment had confirmed the presence of the diverticula with severe diverticulitis and an abscess. The liver lesion was found to be composed of an abscess with a large central necrotic area, and with an intense inflammatory infiltrate composed by lymphoplasma cells and neutrophils, including numerous histiocytes positive for CD68 immunostaining (Figure 1G \& H). We hypothesized that the pathogenetic mechanism underlying NHMH was the transdiaphragmatic chronic irritation due to the hepatic abscess. After 1-year follow-up (clinical and TC) (Figure 1I-L) the patient feels well and without progression of disease.

\section{Discussion}

The first two cases of NHMH were reported by Chan [2]. These lesions looked similar to nodular mesothelial hyperplasia described in hernia sacs and in mesothelial incidental cardiac excrescences, but "as only few cells were positive for cytokeratin and the mesothelial marker HBME1 , whereas most cells were characterized by the histiocytic marker PG-M1 (CD68)," the authors coined the term 'nodular histiocytic/mesothelial hyperplasia' to take into account the marked predominance of histiocytes over mesothelial cells. [2]

NHMH represents an undeniable potential pitfall for pathologists; immunohistochemistry is essential for highlighting the double population of histiocytes and reactive mesothelial cells.

The most challenging aspect of differential diagnoses are with the malignant Langherans cell histiocytosis and with the mesothelioma (mainly with the lymph-histiocytoid type).

Despite the irregular aspect of the nuclei, sometimes with nuclear grooves conferring a 'coffee bean' aspect, as in Langherans cell histiocytosis, the positive immunostaining for CD68 and the negative staining for S100 and CD1a, make this diagnosis unlikely.

On the other hand, the diagnosis of lymphhistiocytoid mesothelioma was also unlikely, due to the absence of necrosis, of mitoses and stromal invasion. Moreover, the negative immunostaining for EMA (usually positive in mesothelial 
neoplastic cells) and p53 (positive in about 30\% of mesotheliomas), together with the low levels of Ki67 positive nuclei was in keeping with a reactive nature of the mesothelial cells.

NHMH probably results from irritation to the mesothelial lining by various causes leading to focal aggregation of histiocytes within retraction pockets or crevices of the serosal cavity. [2]

Recently Rossi et al. observed that NHMH frequently occurs in pleura-damaging processes, such as pneumothorax. [5]

We agree with the hypotesis that $\mathrm{NHMH}$ is a reactive proliferation of histiocytes and mesothelium secondary to chronic pleural irritation.

Nevertheless, in our patient who had no evident pulmonary pathogenic noxa, we hypothesize that a chronic transdiaphragmatic irritation of the pleural mesothelium had occurred, as a consequence of the hepatic, histiocyte rich, abscess, previously resected.

With the aim of supporting this hypothesis, some pathogenetic mechanisms could be cited.

From each side of the diaphragm there are two small areas in which the muscle fibers are missing and replaced by areolar connective tissue. One of these areas contains the superior epigastric branch of the thoracic internal artery and some lymphatic vessels, coming from the abdominal wall and from the convex face of the liver [12]. In our patient, the transdiaphragmatic chronic irritation could have happened via the transdiaphragmatic lymphatic vessels of this small area of the right diaphragm (where the muscle fibers are substituted by areolar tissue) coming from the convex face of the liver, where the subcapsular histiocytes rich abscess was present.

In fact, a unidirectional flow of macrophages from the peritoneal cavity through the diaphragmatic lymphatics to the lung, across the pleural space, has been previously described [13,14].

Furthermore, we would underline that the patient had undergone hemicolectomy for the presence of diverticulitis and bowel abscess. Recently, Parungo et al. [15] demonstrated altered lymphatic drainage in the peritoneal cavity after bowel resection. They observed that the first draining lymph node group was intrathoracic, in the absence of bowel lymphatics, probably through diaphragmatic and anterior chest wall lymphatics. They hypothesized that "patients undergoing bowel resection could have an enhanced lymph flow from the peritoneal space through alternate lymph channels of the diaphragm and parietal surfaces." Actually, we do not know which of the above reported pathogenetic mechanisms were more effective. Perhaps all of them could have had a role in the transdiaphragmatic pleural involvement.

To our knowledge, transdiaphragmatic chronic irritation as pathogenic mechanism underlying $\mathrm{NHMH}$ has never been suggested in the current literature.

In conclusion, we underline the role of VATS to obtain a pathologic diagnosis for chronic pleural effusion to avoid misdiagnosis and unnecessary, potentially dangerous therapies.

\section{Conclusion}

The diagnosis of NHMH is difficult, mainly when no pulmonary noxae are present in the thoracic cavity, as a chronic irritative stimulus. The differential diagnosis with some malignancies (mainly the lymph-histiocytoid mesothelioma) is difficult and it is supported only by histologicy. The awareness of NHMH as a possible consequence of a subdiaphragmatic noxa and the introduction and improvement of new techniques (i.e., in situ hybridization) for the diagnosis of mesothelioma, could make easier the identification and the differential diagnosis of this rare, benign entity.

\section{Financial \& competing interests disclosure}

The authors have no relevant affiliations or financial involvement with any organization or entity with a financial interest in or financial conflict with the subject matter or materials discussed in the manuscript. This includes employment, consultancies, honoraria, stock ownership or options, expert testimony, grants or patents received or pending, or royalties.

No writing assistance was utilized in the production of this manuscript.

4 Chikkamuniyappa S, Herrick J, Jagirdar JS. Nodular histiocytic/mesothelial hyperplasia: a potential pitfall. Ann. Diagn. Pathol. 8(3), 115-120 (2004).

3 Ordonez NG, Ro JY, Ayala AG. Lesions described as nodular mesothelial hyperplasia are primary composed of histiocytes. Am. J.
Rossi G, Cavazza A, Guicciardi N, Marchioni A. Nodular histiocytic/mesothelial hyperplasia on transthoracic biopsy: another Surg. Pathol. 22(3), 285-292 (1998).

potentially mistaken for a neoplasm in

Rosai J, Dehner LP. Nodular mesothelial hyperplasia in hernia sacs: a benign reactive condition simulating a neoplastic process. Cancer 35(1), 165-175 (1975).

2 Chan JK, Loo KT, Yau BK, Lam SY. Nodular histiocytic/mesothelial hyperplasia: a lesion

\section{transbronchial biopsy. Am. J. Surg.}

Pathol. 21(6), 658-663 (1997). 
source of potential pitfall in a lesion frequently present in spontaneous pneumothorax. Histopathology 52(2), 250-252 (2008).

6 Terada T. Immunohistochemical profile of normal mesothelium and histiocytic/ methothelial hyperplasia: a case report. Int. J. Clin. Exp. Pathol. 4(6), 631-636 (2011).

7 Wu M, Anderson A, Kahn LB. A report of mesothelial/monocytic incidental cardiac excrescences and a literature review. Ann. Diagn. Pathol. 4(1), 39-43 (2000).

8 Nicolas MM, Nazarullah A, Jagirdar JS. Nodular histiocytic and mesothelial hyperplasia. Int. J. Surg. Pathol. 19(6), 781-782 (2011).
9 Cai Z, Xie Q, Wang X et al. Nodular histiocytic/mesothelial hyperplasia: a clinicopathologic analysis of 7 cases. Zhonghua Bing Li Xue Za Zhi. 43(4), 256-259 (2014).

10 Veinot JP, Tazelaar HD, Edwards WD, Colby TV. Mesothelial/monocytic incidental cardiac excrescences: cardiac MICE. Mod. Pathol. 7(1), 9-16 (1994).

11 Hu ZL, Lü H, Yin HL, Wen JF, Jin O. A case of mesothelial/monocytic incidental cardiac excrescence and literature review. Diagn. Pathol. 5, 40 (2010).

12 Susan Standring. The anatomical basis of clinical practice. In: Gray's Anatomy (Fortieth Edition). Standring S (Ed.). Elsevier, Oxford, UK (2008).
13 Pitt ML, Anderson AO. Direct transdiaphragmatic traffic of peritoneal macrophages to the lung. Adv. Exp. Med. Biol. 237, 627-632 (1988).

14 Fritz DL, Waag DM. Transdiaphragmatic lymphatic transport of intraperitoneally administered marker in hamsters. Lab. Anim. Sci. 49(5), 522-529 (1999).

15 Parungo CP, Soybel DI, Colson YL et al. Lymphatic drainage of the peritoneal space: a pattern dependent on bowel lymphatics. Ann. Surg. Oncol. 14(2), 286-298 (2007). 